\title{
The meniscus, calcification and osteoarthritis: a pathologic team
}

Paul A MacMullan' and Geraldine M McCarthy*1,2

\begin{abstract}
Articular calcification correlates with osteoarthritis $(\mathrm{OA})$ severity but its exact role in the disease process is unclear. In examining OA meniscal cell function, Sun and colleagues have shown recently that meniscal cells from end-stage $O A$ subjects can generate calcium crystals and that genes involved in calcification are upregulated in OA meniscal cells. Also, this in vitro calcium deposition by OA menisci is inhibited by phosphocitrate. This study should catalyse further work examining the pathological contribution or otherwise of calcium crystals in OA. This would significantly aid the development of potential disease modifying agents in OA, which are currently unavailable.
\end{abstract}

Osteoarthritis (OA) is the leading cause of joint disease in humans. Its complex pathogenesis remains poorly understood but appears multifactorial. OA is slowly progressive and involves all components of the joint, including bone, cartilage, meniscus and synovium. No specific therapy has been identified to reverse or retard the consequences of OA. Therefore, joint replacement surgery is often ultimately the only therapeutic option. It is within this context that the recent work of Sun and colleagues [1] is both novel and clinically relevant.

Calcification of articular cartilage (both hyaline and meniscal) is a well recognized feature of OA and current evidence suggests that it contributes directly to joint degeneration [2]. Calcium-containing crystals are found in more than $60 \%$ of synovial fluid samples from unselected OA patients at knee arthroplasty [3]. Although ample in vitro evidence demonstrates the potent biological effects of calcium-containing crystals, controversy exists as to whether these crystals play a causal role, or are merely a consequence of the joint damage seen in OA [2].

*Correspondence: gmccarthy@rcsi.ie

'Department of Molecular and Cellular Therapeutics, Royal College of Surgeons in Ireland, 123, St Stephen's Green, Dublin 2, Ireland

Full list of author information is available at the end of the article
Calcium pyrophosphate dihydrate (CPPD) and basic calcium phosphate (BCP) are the two most common forms of calcium crystals found in articular cartilage [4]. Their presence is associated with a number of clinical manifestations. For example, CPPD crystals cause acute attacks of articular pseudogout [5] and the presence of intra-articular BCP crystals correlates strongly with the severity of radiographic OA [6]. Both types of crystals are found in OA, but data on the distribution and frequency of BCP crystals vary considerably, mainly due to the lack of simple and reliable methods of detection [7]. Furthermore, the precise source of these crystals is unclear. Recent work clearly demonstrates that $\mathrm{BCP}$ is the predominant crystal type in OA hyaline cartilage, and that chondrocytes derived from $\mathrm{OA}$ hyaline cartilage produce $\mathrm{BCP}$ crystals in vitro [7]. This suggests that cartilage mineralisation with $\mathrm{BCP}$ crystals by chondrocytes is part of the disease process in OA.

While meniscal degeneration and calcification are key features of OA knee joints [8], few studies have investigated the potential role of OA meniscal cells in the pathogenesis of OA. In addressing this neglected area, the work by Sun and colleagues demonstrates a number of key findings. First, calcium crystal deposition is common in the menisci of end-stage OA patients and the pattern of calcification seen is different from that of primary chondrocalcinosis. Secondly, OA meniscal cells, when cultured, induce significantly more calcium deposition than normal control meniscal cells. Thirdly, the expression of genes known to cause articular calcification (ANKH and ENPP1) is upregulated in OA meniscal cells. Finally, calcium deposition by OA meniscal cells is inhibited by phosphocitrate, an observation that is also supported by complementary work using an animal model of OA [9].

In assessing the potential impact of this study by Sun and colleagues, the following methodological weaknesses should be considered. Identification of the specific type of crystals (CPPD or BCP) present in both the clinical samples and the cultured cells was not established. Furthermore, the phenotype of the meniscal cells used in the cultured samples and potential differences between OA meniscal cells and control cells are not addressed. 
Similarly, these cells were not assessed for their ability to produce type X collagen, a recognised marker of chondrocyte hypertrophy and strongly associated with the production of calcium crystals by hyaline cartilage [7]. This is important, as previous work clearly distinguishes specific phenotypes of meniscal cells (with different functional capabilities) in OA menisci compared to normal menisci [10]. An age-related calcification effect also cannot be excluded on the basis of the relatively small sample sizes used. Finally, as correctly stated by the authors, the findings of the study do not prove a causal role for calcium crystal deposition in OA.

Nonetheless, meniscal calcification, mediated by meniscal cells, is a potentially important contributory factor in the pathogenesis of OA. Therefore, notwithstanding the limitations noted above, the clinical relevance of this study is timely. To truly test the hypothesis that calcium crystals play a causative role in OA, animal studies in which these crystals (especially BCP) are injected intra-articularly are warranted [2]. Should such studies demonstrate the induction or acceleration of joint degeneration that could then be arrested or reversed by an agent such as phosphocitrate, this would provide proof-of-concept evidence for the pathogenicity or otherwise of these crystals in OA.

A major barrier to developing medical interventions in $\mathrm{OA}$ has been the reliance on end-stage radiographic outcome measures, which often take years to develop and are therefore unsuitable for placebo-controlled trials. The adoption of articular cartilage calcification as a surrogate marker of OA disease, coupled with the development of improved detection methods for BCP crystals, could enable trials of targeted anti-crystal therapies with biological endpoints and a fast turnaround time. This could significantly advance the search for an effective medical intervention in the most common of human joint disorders.

Abbreviations

$\mathrm{BCP}=$ basic calcium phosphate; $\mathrm{CPPD}=$ calcium pyrophosphate dihydrate; $\mathrm{OA}=$ osteoarthritis
Competing interests

The authors declare that they have no competing interests.

\section{Author details}

'Department of Molecular and Cellular Therapeutics, Royal College of Surgeons in Ireland, 123, St Stephen's Green, Dublin 2, Ireland. 2Division of Rheumatology, Mater Misericordiae University Hospital and University College

Dublin, Eccles St, Dublin 7, Ireland

Published: 20 May 2010

References

1. Sun Y, Mauerhan DR, Honeycutt PR, Kneisl JS, Norton HJ, Zinchenko N, Hanley EN Jr, Gruber HE: Calcium deposition in osteoarthritic menisci and meniscal cell culture. Arthritis Res Ther, 12:R56.

2. McCarthy GM, Cheung HS: Point: Hydroxyapatite crystal deposition is intimately involved in the pathogenesis and progression of human osteoarthritis. Curr Rheumatol Rep 2009, 11:141-147.

3. Derfus BA, Kurian JB, Butler JJ, Daft LJ, Carrera GF, Ryan LM, Rosenthal AK: The high prevalence of pathologic calcium crystals in pre-operative knees. J Rheumato/ 2002, 29:570-574.

4. Molloy ES, McCarthy GM: Calcium crystal deposition diseases: update on pathogenesis and manifestations. Rheum Dis Clin North Am 2006, 32:383-400, vii.

5. McCarty DJ: Crystal-induced inflammation of the joints. Annu Rev Med 1970, 21:357-366.

6. McCarthy GM: Inspirational calcification: how rheumatology research directs investigation in vascular biology. Curr Opin Rheumatol 2009, 21:47-49.

7. Fuerst M, Bertrand J, Lammers L, Dreier R, Echtermeyer F, Nitschke Y, Rutsch F, Schäfer FK, Niggemeyer O, Steinhagen J, Lohmann $\mathrm{CH}$, Pap T, Rüther W: Calcification of articular cartilage in human osteoarthritis. Arthritis Rheum 2009, 60:2694-2703.

8. Bennett LD, Buckland-Wright JC: Meniscal and articular cartilage changes in knee osteoarthritis: a cross-sectional double-contrast macroradiographic study. Rheumatology (Oxford) 2002, 41:917-923.

9. Cheung HS, Sallis JD, Demadis KD, Wierzbicki A: Phosphocitrate blocks calcification-induced articular joint degeneration in a guinea pig model. Arthritis Rheum 2006, 54:2452-2461.

10. Verdonk PC, Forsyth RG, Wang J, Almqvist KF, Verdonk R, Veys EM, Verbruggen $\mathrm{G}$ : Characterisation of human knee meniscus cell phenotype. Osteoarthritis Cartilage 2005, 13:548-560.

doi:10.1186/ar2993

Cite this article as: MacMullan PA, McCarthy GM: The meniscus, calcification and osteoarthritis: a pathologic team. Arthritis Research \& Therapy 2010, 12:116. 